\title{
Anisakiasis en una paciente portadora de una pequeña hernia hiatal. Caso clínico
}

\author{
Rubén Mercado Pla, Patricio Torres $\mathrm{H}^{2 a}$, Luis Carlos Gil L33, \\ Luis G oldin G ${ }^{4}$.
}

\section{Anisakiasis in a patient with a small hiatal hernia. Report of one case}

We describe the presence of anisakiasis in a patient who had a small hiatal hernia. A 60 year-old women presented general malaise, burning pain, flatulence, persistent nausea and abdominal distension during five days before consulting. She referred that she ate a dish of marinated raw fish with lemon and pepper ("cebiche") and after a few hours the symptoms began. An esophagogastroscopy showed a white colour larva of approximately three $\mathrm{cm}$ with the cephalic end partially adhered and localized in the mucosa of the hiatal hernia. During the procedure the worm was easily extracted. The morphologic study of the specimen identified it as a stage IV larva of Pseudoterranova sp. The clinical condition of the patient improved after the extraction of the parasite (Rev Méd Chile 2006; 134: 1562-4).

(Key words: Anisakiasis; Nematoda; Gastroscopy)

\begin{abstract}
Recibido el 12 de septiembre, 2005. Aceptado el 29 de mayo, 2006.
${ }^{1}$ Unidad Docente de Parasitología, Facultad de Medicina, Universidad de Chile. ${ }^{2}$ Instituto de Parasitología, Facultad de Medicina, Universidad Austral de Chile. ${ }^{3}$ Departamento de Gastroenterología, Hospital Clínico, Universidad de Chile. ${ }^{4}$ Gastroenterología, Clínica Dávila. Santiago de Chile.

aTecnólogo Médico. Ph.D. en Parasitología
\end{abstract}

L

a anisakiasis humana es causada por la parasitación de larvas de nematodos de la familia Anisakidae, principalmente por especies de los géneros Anisakis (anisakiosis) o Pseudoterranova (pseudoterranovosis) $^{1}$. En Chile, el primer caso fue descrito por Sapunar y cols en $1976^{2}$. La anisakiasis se adquiere mediante el consumo de pescado crudo (cebiche, sushi), ahumado en frío, 0 insuficientemente cocido, que contenga las larvas del tercer estadio de estos nematodos de

Correspondencia a: Rubén Mercado. Las Palmeras 299, Interior Quinta Normal. Santiago, Chile. Fono 56-2-6817911. Fax: 56-2-6814499. E mail: rmercado@med.uchile.cl origen marino ${ }^{3-6}$. Las larvas, al ingresar al organismo, pueden parasitar preferentemente estómago o localizarse en duodeno, yeyuno, íleon o colon, así como también en el esófago ${ }^{7}$. Las larvas pueden penetrar completamente la pared gastrointestinal y alcanzar pulmones, hígado, páncreas u ovario. La localización esofágica es muy poco frecuente, correspondiendo a 0,8\% de 2.514 casos publicados hasta 1990 en Japón, país que registra la mayor casuística de anisakiasis en el mundo ${ }^{7,8}$.

El objetivo de esta comunicación es dar a conocer la parasitación de una paciente por una larva de cuarto estadio de Pseudoterranova sp. El parásito estaba localizado en la mucosa de una pequeña hernia hiatal. 
Caso clínico. Paciente de 60 años de edad, sexo femenino, dueña de casa, nacida y residente de Santiago, con historia de reflujo gastroesofágico y hernia hiatal diagnosticada dos años antes de su consulta, por endoscopia. Consultó a gastroenterólogo por pirosis, malestar general, náusea persistente de cinco días de evolución, la que asociaba a distensión abdominal y flatulencia. Se realizó endoscopia digestiva alta en la cual se describió: esófago: línea $\mathrm{Z}$ a $36 \mathrm{~cm}$ de la arcada dentaria, 1-2 $\mathrm{cm}$ sobre el hiato. La mucosa del tercio distal se observaba engrosada y blanquecina. Pliegues gástricos estaban algo engrosados sobrepasaban el hiato hacia proximal. Bajo la línea $\mathrm{Z}$, en la mucosa, se observaba pequeño verme parcialmente adherido por su extremo cefálico, que se extrajo con pinza de biopsia. Estómago: Cuerpo normal. El antro presentaba erosiones planas y verrucosas. El duodeno: bulbo y segunda porción no mostraron lesiones.

Se diagnosticó: esofagitis crónica, hernia hiatal pequeña, zanisakiasis?, gastritis erosiva moderada.

El verme no se encontraba inmerso en la mucosa esofágica, pero se pudo apreciar que estaba ligeramente adherido e ingresaba por su extremo cefálico a la hernia hiatal. Era único e impresionaba estar vivo.

Reinterrogada, la paciente explicó que sus síntomas se incrementaron luego de un viaje a la localidad de Limache, donde en un negocio casero ingirió cebiche. Después de algunas horas presentó gran intensidad de síntomas, los que persistieron hasta cinco días después, cuando acudió a la consulta médica donde se le extrajo el gusano. La extracción generó una rápida mejoría de los síntomas. Se prescribió tratamiento con mebendazol.

La larva del parásito fue fijada en etanol de $70 \%$. Posteriormente, en el laboratorio fue diafanizada en lactofenol para observar sus estructuras e identificarla. Presentó las siguientes características morfológicas (dimensiones en $\mathrm{mm}$ ): tres labios en la región cefálica y sin diente perforador, no se observó mucrón en la región caudal; longitud corporal: 25,7; anchura máxima: 0,7; longitud del esófago: 1,6; longitud del ventrículo: 0,9; longitud de ciego intestinal: 0,9; cola: 0,2; distancia anillo nervioso-extremo anterior: 0,4.

El espécimen fue depositado en la colección del Instituto de Parasitología de la Universidad Austral de Chile (IPUAT № 286).

\section{COMENTARIO}

Esta comunicación se refiere a la descripción de un nuevo paciente con pseudoterranovosis en Chile, esta vez, una enferma que presentaba una pequeña hernia hiatal. Entre las anisakiasis humanas, la pseudoterranovosis es menos frecuente que la anisakiosis a nivel mundial ${ }^{7}$. Sin embargo, en Chile, la mayor casuística corresponde a pseudoterranovosis, lo cual puede estar relacionado con la frecuencia de parasitación de la musculatura de los pescados marinos que más frecuentemente consumen las personas ${ }^{5,6}$. Al menos, un estudio preliminar en cinco especies de pescados marinos habitualmente comercializados para su consumo, mostró una mayor frecuencia e intensidad de infección por larvas de Pseudoterranova, que de otros anisakidos ${ }^{6}$. Lo anterior también depende en parte de la magnitud de las poblaciones y de las migraciones de los mamíferos marinos que actúan como huésped definitivo natural de dichos nematodos ${ }^{8}$. En Chile, las larvas recolectadas en la mayoría de los casos humanos han sido identificadas morfológicamente como Pseudoterranova sp o Pseudoterranova decipiens (sensu stricto) ${ }^{5}$. Una nueva especie, Pseudoterranova cattani fue descrita en lobos marinos (Otarya byronia) en las costas chilenas ${ }^{9}$, la que corresponde a una de las ocho especies conocidas en el género y que también ha sido registrada en cuatro especies de peces, mediante pruebas moleculares $^{10}$.

Las larvas de Pseudoterranova spp serían menos invasoras de la pared del tracto digestivo que las larvas de Anisakis spp ${ }^{7}$. El patrón de la pseudoterranovosis humana en Chile es semejante al comunicado en casos descritos en los Estados Unidos de Norteamérica, donde, por lo general, no hay penetración tisular de las larvas, las que finalmente son eliminadas por la boca o junto con las heces de los pacientes ${ }^{2-5,11}$. En el presente caso, la hernia hiatal de la paciente pudo haber contribuido a la permanencia de la larva en dicha zona.

Una adecuada cocción de la carne de pescado, con temperaturas sobre $63^{\circ} \mathrm{C}$ por $15 \mathrm{~s}$, o la congelación a $-20^{\circ} \mathrm{C}$ por siete días, puede prevenir la infección humana por nematodos anisaki$\operatorname{dos}^{6}$. 


\section{REFERENCIAS}

1. Kassai T, Cordero Del Campillo M, Euzeby J, Gaafar S, Hiepe T, Himonas CA. Standardized nomenclature of animal parasitic diseases (SNOAPAD). Veterinary Parasitology 1988; 29: 299-326.

2. Sapunar J, Doerr E, Letonja T. Anisakiasis humana en Chile. Bol Chil Parasitol 1976; 31: 79-83.

3. Apt W, Hisamoto T, Lorens P, Alcaíno H. Anisakiasis gástrica en Chile. Rev Méd Chile 1980; 108: 825-7.

4. Mercado R, Torres P, Maira J. Human case of gastric infection by fourth larval stage of Pseudoterranova decipiens (Nematoda, Anisakidae). Rev Saúde Pub 1997; 31: 131-3.

5. Mercado R, Torres P, Muñoz V, Apt W. Human infection by Pseudoterranova decipiens in Chile. Report of seven cases. Mem Inst Oswaldo Cruz 2001; 96: 653-5.

6. Torres P, Moya R, Lamшa J. Nematodos anisakidos de interés en salud pública en peces comercializados en Valdivia, Chile. Arch Med Vet 2000; 32: 107-13.
7. IshikuRa $\mathrm{H}$, KikUChi $\mathrm{K}$, Nagasawa $\mathrm{K}$, Ooiwa $\mathrm{T}$, TaKamiYa H, Sato N et al. Anisakidae and anisakidosis. In: Sun T. Progress in Clinical Parasitology. Springer-Verlag, New York, USA, 1993; pp. 43102.

8. TAKAHASHI S, IshiKURA $H$, KIKUCHI K. Anisakiasis: global point of view. In: Host response to international parasitic zoonoses. Ishikura $\mathrm{H}$, Aikawa M, Itakura H. Kikuchi K (eds.) Springer- Verlag, Tokyo, Japón, 1998; pp. 109-120.

9. George-Nascimento M, Lianos A. Pseudoterranova cattani sp. nov. (Ascaridoidea: Anisakidae), a parasite of the South American sea lion Otaria byronia De Blainville from Chile. Revista Chilena Historia Natural 2000; 73: 93-8.

10. McCleluand G. The trouble with sealworms (Pseudoterranova decipiens species complex, Nematoda): a review. Parasitology 2002; 124: S183-S203.

11. Amin OM, EIdelman WS, Domke W, Bailey J, Pfeifer G. An unusual case of anisakiasis in California, USA. Comp Parasitol 2000; 67: 71-5. 